\title{
The growth and thermodynamical feasibility of tungsten diselenide single crystals using chemical vapour transport technique
}

\author{
K SUNIL and M A ITTYACHEN* \\ School of Pure and Applied Physics, Mahatma Gandhi University, Priyadarshini \\ Hills P. O., Kottayam 686560 , India \\ MS received 22 February 1996; revised 23 December 1996

\begin{abstract}
Thermodynamical feasibility study and the growth of layer structured transition metal dichalcogenide single crystals of $\mathrm{WSe}_{2}$, using iodine as transporting agent, has been reported in this paper. The characterization of the grown samples have been done by $\mathrm{X}$-ray analysis.
\end{abstract}

Keywords. Transition metal dichalcogenide; tungsten diselenide; chemical vapour transport; thermodynamical feasibility.

\section{Introduction}

The transition metal disulphides and diselenides have attracted considerable attention during the past few years (Legma et al 1991; Patel 1992; Agarwal et al 1993; Gallagher et al 1994). These $\mathrm{MX}_{2}(\mathrm{M}=$ transition metal; $\mathrm{X}=$ chalcogen $)$ compounds are formed by a layer of transition metal atoms between two layers of sulphur or selenium atoms. There is a strong covalent bonding within the layers, but a weak Van der Waals bonding between them. These materials are used in such diverse applications as catalysis, batteries and lubricants. Recently, these materials have found potential use in the fabrication of photoelectrochemical (PEC) solar cells for solar energy conversion (Gobrecht et al 1978; Fan et al 1980; Tributsch 1980). It has also been realized that selenides are more efficient as solar cell materials than sulphides (Canfield et al 1981). In the light of this realization, the growth of tungsten diselenide single crystals using chemical vapour transport technique and the fabrication of PEC solar cells have attracted more attention recently (Agarwal et al 1989a). The PEC cell characteristics have however not reached up to the predicted level due to the lack of good quality crystals. Theoretical thermodynamical investigations may help to grow these crystals with better characteristics.

In the method of chemical vapour transport, an exploratory work is needed to avoid the ineffective and time consuming empirical trial and error fashion for the transport of a particular material. In many cases the models (Seaki 1967; Richardson 1978; Wolf et al 1978; Benard et al 1981; Nolang 1983) allow one to predict the composition of solids to be deposited in the crystallization zone or to determine the pre-conditions for the deposition of solid(s) of desired composition. It is also obvious that the theoretical models (Emmenegger 1968; Wehmeier 1970) are not adequate to explain the successful growth of binary as well as ternary chalcogenides taking the constituent elements in the stoichiometric ratio as the source material. Nitsche (1975) and Paorici et al (1982) established that the constituent elements could be employed as the starting materials.

* For correspondence 
Vengatesan et al (1986) proposed a thermodynamical analysis of chemical vapour transport of ternary chalcogenides taking the elements as the source materials. Prediction of minimum source temperature $\left(T_{\mathrm{s}}\right)$, minimum growth temperature $\left(T_{\mathrm{d}}\right)$ and the influence of temperature and the transporting agent concentration on growth are successfully done on the basis of this model. In the present work, an attempt has been made to consider the growth and thermodynamical analysis of the layered structured binary compound tungsten diselenide using iodine as the transporting agent.

\section{Model and calculations results}

The constituent elements tungsten and selenium are taken in stoichiometric proportions along with the transporting agent, -iodine- at the source end. They react to form the gaseous binary iodide and selenium at high temperature. This gaseous binary iodide diffuses into the colder growth zone due to drop in temperature. At the growth zone, the binary iodide plus selenium react back to form the binary chalcogenide with the release of iodine. The iodine diffuses back to the source end to form the metal iodide once again. This process is represented by the equation

$$
\mathrm{W}(s)+2 \mathrm{Se}(s)+\mathrm{I}_{2}(g) \stackrel{T_{\mathrm{s}}}{\rightleftharpoons} \mathrm{WI}_{2}(g)+\operatorname{Se}_{2}(g) \stackrel{T_{\mathrm{d}}}{\rightleftharpoons} \mathrm{WSe}_{2}(s)+\mathrm{I}_{2}(g)
$$

The transport of the binary compound is feasible only if the Gibb's free energy change $\Delta G$ of the reaction is negative.

The hypothetical transport reaction is

$$
\mathrm{W}(s)+\mathrm{Se}(s)+\mathrm{I}_{2}(g) \rightleftharpoons \mathrm{WI}_{2}(g)+1 / 2 \mathrm{Se}_{2}(g) .
$$

The general $\Delta G$ function is

$$
\Delta G=\Delta G^{\circ}+R T \ln K_{\mathrm{p}} .
$$

where $\Delta G^{\circ}$ is the standard free energy change, $R$, the universal gas constant, $T$, the absolute temperature, and $K_{\mathrm{p}}$, the equilibrium constant.

The formation of the binary compound from binary iodide and selenium can be represented by the equation

$$
\mathrm{WI}_{2}(g)+\mathrm{Se}_{2}(g) \rightleftharpoons \mathrm{WSe}_{2}(s)+\mathrm{I}_{2}(g) \text {. }
$$

Free energy function for (1a)

$$
\Delta G_{1 \mathrm{a}}=\Delta G_{1 \mathrm{a}}^{\circ}+R T \ln K_{\mathrm{p} 1 \mathrm{a}},
$$

where the equilibrium constant,

$$
K_{\mathrm{p} 1 \mathrm{a}}=\frac{\mathrm{P}_{1 \mathrm{a}}\left(\mathrm{I}_{2}\right)}{\mathrm{P}_{1 \mathrm{a}}\left(\mathrm{WI}_{2}\right)^{2}},
$$

where $P_{1 a}\left(I_{2}\right)$ and $P_{1 a}\left(W I_{2}\right)$ are the partial pressures of the iodine and the binary iodide. From the stoichiometry of (1),

$$
P_{1 \mathrm{a}}\left(\mathrm{WI}_{2}\right)=\mathrm{P}_{1 \mathrm{a}}\left(\mathrm{Se}_{2}\right)
$$


where $\mathrm{P}_{1 \mathrm{a}}\left(\mathrm{Se}_{2}\right)$ is the partial pressure of selenium.

At equilibrium, $\Delta G_{1 \mathrm{a}}=0$.

i.e. $\quad-\Delta G_{1 \mathrm{a}}^{\circ}=R T\left[\ln \mathrm{P}_{1 \mathrm{a}}\left(\mathrm{I}_{2}\right)-2 \ln \mathrm{P}_{1 \mathrm{a}}\left(\mathrm{WI}_{2}\right)\right]$.

Similarly for (2)

and

$$
\Delta G_{2}=\Delta G_{2}^{\circ}+R T \ln K_{\mathrm{p}_{2}}
$$

$$
\Delta G_{2}=\Delta G_{2}^{\circ}+R T\left[3 / 2 \ln \mathrm{P}_{2}\left(\mathrm{WI}_{2}\right)-\ln \mathrm{P}_{2}\left(\mathrm{I}_{2}\right)\right],
$$

since (3) is also valid for (4b).

By combining (5) and (4a)

$$
\Delta G_{2}=\Delta G_{2}^{\circ}+0.75 \Delta G_{1 \mathrm{a}}^{\circ}-0.25 \ln R T \mathrm{P}_{2}\left(\mathrm{I}_{2}\right) .
$$

Since heat capacity functions are not available, $\Delta G^{\circ}$ function is evaluated from $\Delta H^{\circ}$ and $\Delta S^{\circ}$. The standard thermodynamic datas for (6) are listed in table 1.

$$
\Delta G_{2}=-50205+40 T-0.495 T \ln \mathrm{P}\left(\mathrm{I}_{2}\right) .
$$

It is found that $\Delta G_{2}$ is negative at any temperature below $1177^{\circ} \mathrm{K}$ for iodine concentration of $5 \mathrm{mg} / \mathrm{cm}^{3}$. So the source temperature $\left(T_{\mathrm{s}}\right)$ should be kept below $1177^{\circ} \mathrm{K}$ for effective transport.

The dependence of $\Delta G_{2}$ on the amount of iodine at any constant temperature is represented in figure 1 . The transport of binary compound is more feasible at lesser concentration of iodine as revealed by the negative $\Delta G_{2}$ at much lower temperature of $1148^{\circ} \mathrm{K}$. Since the partial pressure is in the logarithmic term, the influence of partial pressure of iodine is comparatively less than that of temperature. The variation of $\Delta G_{2}$ on temperature for fixed iodine concentration is also represented in figure 2. From figure 2 , it is evident that sign of $\Delta G_{2}$ changes at a particular temperature for fixed iodine concentration.

Due to the fixed temperature gradient between the hot zone and cold zone, the binary iodide and selenium diffuse to the colder zone. At the colder zone two types of reactions are possible.

$$
\begin{aligned}
& \mathrm{WI}_{2}(g)+\mathrm{Se}_{2}(g) \mathrm{W}(s)+2 \mathrm{Se}(s)+\mathrm{I}_{2}(g), \\
& \mathrm{WI}_{2}(g)+\mathrm{Se}_{2}(g) \rightleftharpoons \mathrm{WSe}_{2}(s)+\mathrm{I}_{2}(g) .
\end{aligned}
$$

Equations (8) and (9) are for elemental formation and material formation, respectively.

Table 1. Thermodynamic datas.

\begin{tabular}{lccc}
\hline & $H f^{\circ}(\mathrm{cal} / \mathrm{mol})$ & $S^{\circ}(\mathrm{cal} / \mathrm{mol})$ & \multicolumn{1}{c}{ Refs. } \\
\hline $\mathrm{W}(s)$ & 0.0000 & 7.80 & (Weast 1987) \\
$\mathrm{Se}(s)$ & 0.0000 & $10 \cdot 144$ & (Weast 1987) \\
$\mathrm{I}_{2}(g)$ & +14923 & $62 \cdot 28$ & (Wagman et al 1968) \\
$\mathrm{Se}_{2}(g)$ & +34900 & 60.20 & (Wagman et al 1968) \\
$\mathrm{WI}_{2}(g)$ & -16000 & $-32 \cdot 00$ & (Rolsten 1961) \\
$\mathrm{WSe}_{2}(s)$ & -45000 & 21.50 & (Mills 1974) \\
\hline
\end{tabular}




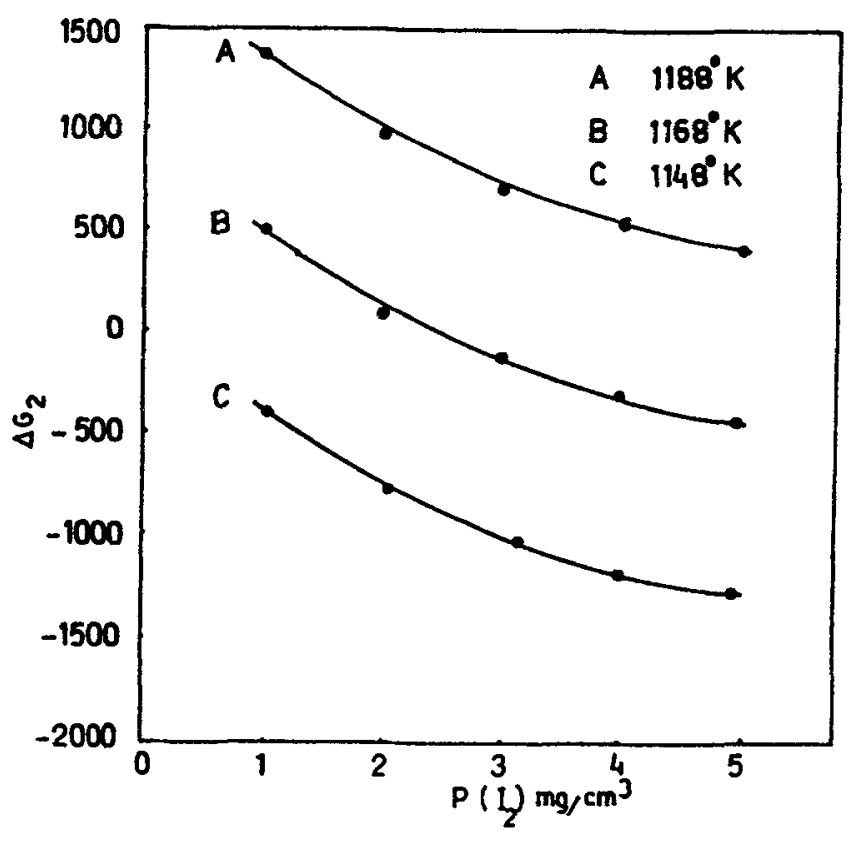

Figure 1. Dependence of $\Delta G$ on iodine concentration at various temperatures.

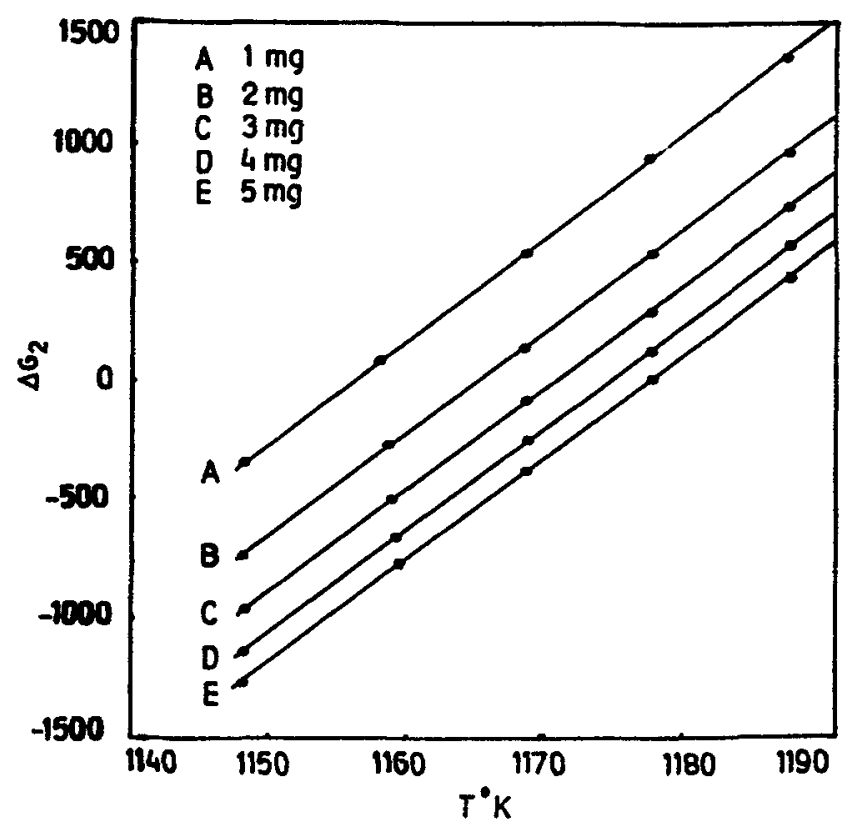

Figure 2. Variation of $\Delta G$ with temperature for different iodine concentrations.

For the effective transport, assume $K_{\mathrm{p}}=1$ for both equations. Then the free energy functions $\Delta G_{8}$ and $\Delta G_{9}$ are

$$
\begin{aligned}
& \Delta G_{8}=\Delta H f^{\circ}{ }_{8}-T \Delta S^{\circ}{ }_{8}, \\
& \Delta G_{9}=\Delta H f^{\circ}{ }_{9}-T \Delta S^{\circ}{ }_{9} .
\end{aligned}
$$


By using thermodynamic data of table 1 ,

$$
\begin{aligned}
& \Delta G_{8}=-3977-6 \cdot 116 T, \\
& \Delta G_{9}=-48977-55.58 T .
\end{aligned}
$$

These equations show that the formation of the element as well as material is possible at any temperature.

Take the ratio of the two equations as

$$
\left[\frac{\Delta G_{9}}{\Delta G_{8}}\right]_{T}=10 \cdot 28,
$$

it is found that for a constant temperature, the ratio is $10-28$. It shows that the feasibility for (11) is $10 \cdot 28$ times higher than that for (10).

Theoretical thermodynamical calculations have shown that reaction is possible in order to have a stable $\mathrm{WSe}_{2}$ phase as the growth zone. In order to verify this detailed experiments were conducted.

\section{Experimental}

Single crystals of tungsten diselenide have been grown by many workers (Al-Hilli and Evans 1972; Agarwal and Rao 1989; Agarwal et al 1989b; Legma et al 1993) using different kinds of transporting agents. Since the iodine possesses the least bond energy among the halogens (Emeleus and Sharpe 1973), attention has been given to grow $\mathrm{WSe}_{2}$ using iodine as transporting agent. The growth parameters selected in the present investigation are different from those used by other workers (Al-Hilli and Evans 1972).

99.99\% Pure tungsten and selenium powders obtained from Merk were taken in stoichiometric proportions and were placed in transparent quartz ampoules (length $=200 \mathrm{~mm}$ and diameter $=24 \mathrm{~mm}$ ) which were etched and vacuum baked. The total charge used in each experiment was $10 \mathrm{~g}$. The transporting agent iodine $\left(5 \mathrm{mg} / \mathrm{cm}^{3}\right)$ was also placed in the quartz ampoule along with the powders. The ampoule was evacuated and sealed at a pressure of $10^{-5}$ torr. Thoroughly shakened ampoule was placed in the two zone horizontal furnace having appropriate temperature profile.

The temperature of the furnace was increased slowly in steps of $343^{\circ} \mathrm{K} / \mathrm{h}$. The temperature of the vapourization and deposition zones were $T_{\mathrm{s}}=1173^{\circ} \mathrm{K}$ and $T_{d}=1103^{\circ} \mathrm{K}$, respectively. After five days of uninterrupted heat treatment, single crystals of $\mathrm{WSe}_{2}$ in platelets form with c-axis normal to the plane of the plates were obtained. The separated crystals were observed under metallurgical microscope in order to ascertain the growth nature. The surface of the platelets were highly reflective and devoid of any bulk defects. In vapour transport mechanism, density of the vapour species need not to be distributed uniformly throughout the capsule due to the local variations in supersaturation. The condition was quite critical at the growth region. The spiral growth usually occurred at lower supersaturation of the species and layer growth initiated at higher supersaturation (Elwell and Scheel 1975; Krishan Lal 1982). In this particular case these two mechanisms occurred at 


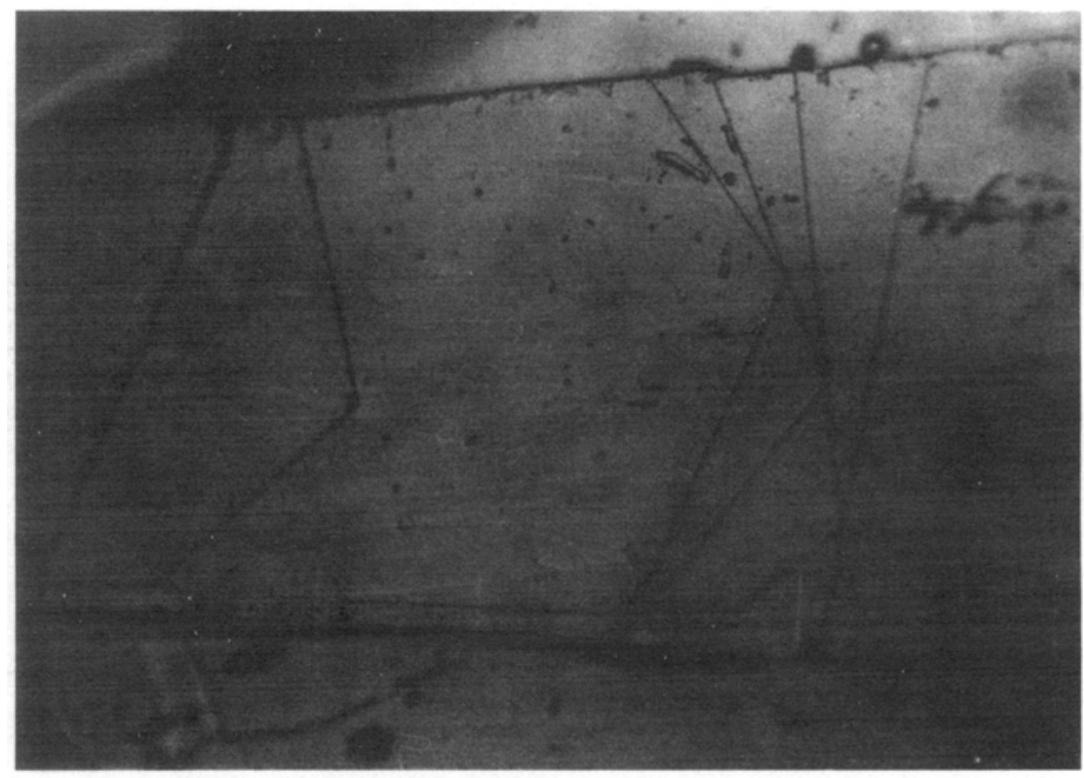

Figure 3. Growth layers.

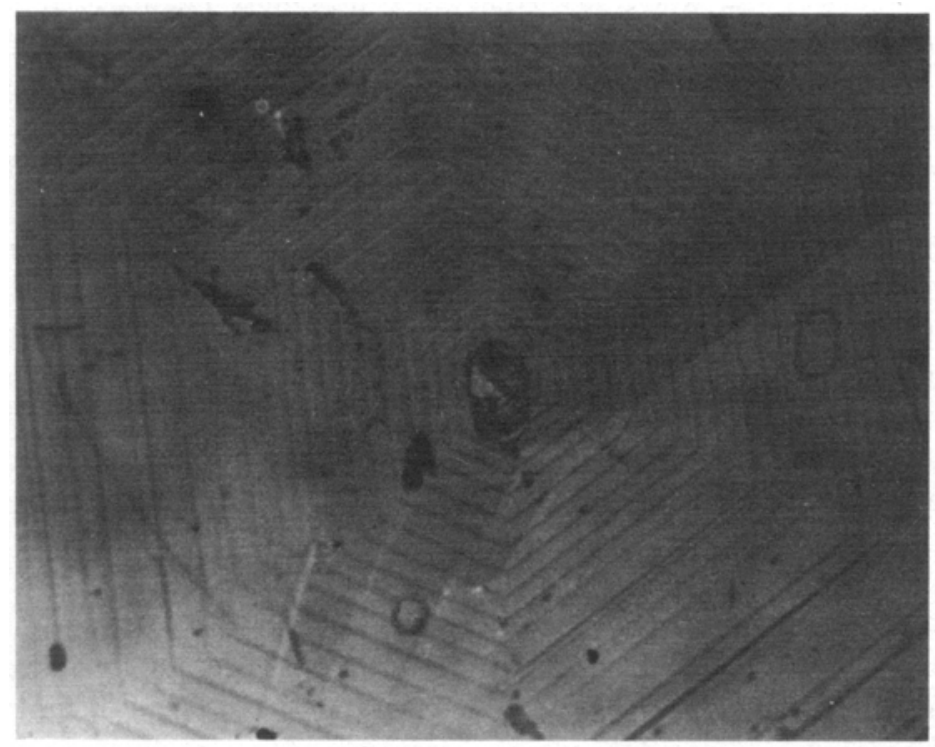

Figure 4. Growth spiral.

micro regions in the growth locations. Hence both these types of growth were expected. Figure 3 is an example for two dimensional nucleation and figure 4 , growth by spiral mechanisms. It was found that $90 \%$ of the crystals were grown by former and remaining ones by latter. 


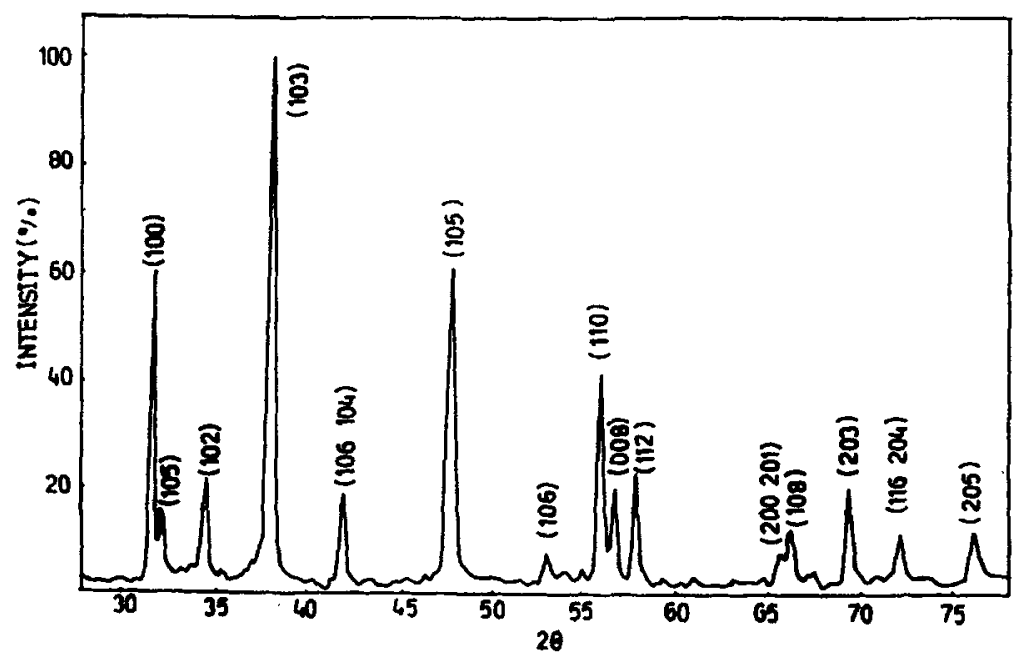

Figure 5. X-ray diffractogram.

\section{Characterization}

Figure 5 shows the diffractogram of the grown sample using $\mathrm{Cu} \mathrm{K} \alpha$ radiation from Shimadzu XD-610 diffractometer. From the XRD peaks, the lattice parameters measured were $a=3.29 \AA, c=12.97 \AA$ which were consistent with literature values (Glemser 1948).

\section{Conclusion}

Preliminary thermodynamical calculations show that for efficient transport of binary iodide, the temperature of the hot zone should be around $1177^{\circ} \mathrm{C}$. The formation of $\mathrm{WSe}_{2}$ and its constituent elements have equal probability at wide range of temperatures. The calculations show that the possibility of formation of $\mathrm{WSe}_{2}$ is $10 \cdot 28$ times higher than that of the formation of the elements in the deposition zone. On this basis the temperature of the growth zone has been assigned to $1103^{\circ} \mathrm{K}$ for a temperature gradient, $T=347^{\circ} \mathrm{K}$.

Detailed experiments have been conducted by changing the temperature of growth zone and source zone in order to see the best condition for the formation of good WSe crystals. Platelet single crystals are formed when the temperature zones are fixed near theoretical values. Our results also show that the lower iodine concentration reduces contamination of the crystals. These crystals were characterized using X-ray method. The examination of the crystals showed that the growth is predominantly taking place due to spiral mechanisms, two dimensional nucleation and spreading of layers.

\section{References}


Agarwal M K, Rao V V and Pathak V M 1989b J. Cryst. Growth 97675

Al-Hilli A A and Evans B L 1972 J. Cryst. Growth 1593

Benard C, Constant G and Feurer R 1981 J. Electrochem. Soc. Solid State Sci. Technol. 1282447

Canfield D, Kam K K, Kline G and Parkinson B A 1981 Solar Energy Mater. 4301

Elwell D and Scheel H J 1975 Crystal growth from high-temperature solutions (London: Academic Press Inc.) p. 157

Emeleus H J and Sharpe A G 1973 Modern aspects of inorganic chemistry (London: ELBS Edition)

Emmenegger F 1968 J. Cryst. Growth 3/4 135

Fan F R, White H S, Wheeler B and Bard A J 1980 J. Electrochem. Soc. 127518

Gallagher M J, Todd G Ruskell, Dong Chen, Dror Sarid and Howard Jenkinson 1994 Appl. Phys. Lett. 64 256

Glemser 1948 Z. Anorg. Chemie. 257241

Gobrecht J, Gerischer H and Tributsch H 1978 J. Electrochem. Soc. 1251086

Krishan Lal (ed) 1982 Synthesis, crystal growth and characterization (Amsterdam, New York, Oxford: North-Holland Publishing Company) p. 2

Legma J B, Vacquier G, Traor'e H and Casalot A 1991 Mater. Sci. Engg. B8 167

Legma J B, Vacquier G and Casalot A 1993 J. Cryst. Growth 130253

Mills K C 1974 Thermodynamic data for inorganic sulphides, selenides and tellurides (London: Butterworths)

Nitsche R 1975 J. Phys. 36 C3.9

Nolang B 1983 Application of equilibrium computations to CVT and related systems, Acta University, Upsaliensis, No. 691

Paorici C, Zanotti L and Curti M 1982 Cryst. Res. Tech. 17917

Patel S G 1992 Cryst. Res. Technol. 27285

Richardson M W 1978 Theoretical and practical aspects of chemical vapour transport as a crystal growth method, Doctoral Thesis, Acta University, Upsaliensis, No. 464

Rolsten R F 1961 Iodide metals and metal iodides (New York-London: John Wiley and Sons. Inc.)

Seaki M 1967 J. Cryst. Growth 3677

Tributsch H 1980 J. Appl. Phys. 2361

Vengatesan B, Kanniah V N and Ramasamy P 1986 J. Mater. Sci. Lett. 5984

Wagman D D, Evans W H, Parker V B, Halow I, Bailey S M and Schumm R H 1968 NBS Technical Note 270-3, United States, Dept. of Commerce, National Bureau of Standards

Weast R C 1987 CRC Hand Book of Chemistry and Physics (Inc. Boca Raton Florida: CRC Press) 1st Students Edition

Wehmeier F H 1970 J. Cryst. Growth 6341

Wolf E, Oppermann H, Krabbes G and Reichelt W 1978 Current topics in material science (ed) E Kaldis (Amsterdam: North-Holland) Vol. 1 